\title{
Properties of the water to boron nitride interaction: From zero to two dimensions with benchmark accuracy
}

Yasmine S. Al-Hamdani, , Mariana Rossi, , Dario Alfè, , Theodoros Tsatsoulis, , Benjamin Ramberger, , Jan Gerit Brandenburg, , Andrea Zen, , Georg Kresse, , Andreas Grüneis, , Alexandre Tkatchenko, and , and Angelos Michaelides

Citation: The Journal of Chemical Physics 147, 044710 (2017); doi: 10.1063/1.4985878

View online: http://dx.doi.org/10.1063/1.4985878

View Table of Contents: http://aip.scitation.org/toc/jcp/147/4

Published by the American Institute of Physics

\section{Articles you may be interested in}

Nanolayering around and thermal resistivity of the water-hexagonal boron nitride interface

The Journal of Chemical Physics 147, 044709 (2017); 10.1063/1.4985913

Structural and dynamic characteristics in monolayer square ice

The Journal of Chemical Physics 147, 044706 (2017); 10.1063/1.4995432

Hydrogen bonding and vibrational energy relaxation of interfacial water: A full DFT molecular dynamics simulation

The Journal of Chemical Physics 147, 044707 (2017); 10.1063/1.4995437

Perspective: A controversial benchmark system for water-oxide interfaces: $\mathrm{H}_{2} \mathrm{O} / \mathrm{TiO}_{2}(110)$

The Journal of Chemical Physics 147, 040901 (2017); 10.1063/1.4996116

Extension of the D3 dispersion coefficient model

The Journal of Chemical Physics 147, 034112 (2017); 10.1063/1.4993215

Drying and wetting transitions of a Lennard-Jones fluid: Simulations and density functional theory

The Journal of Chemical Physics 147, 044701 (2017); 10.1063/1.4993515

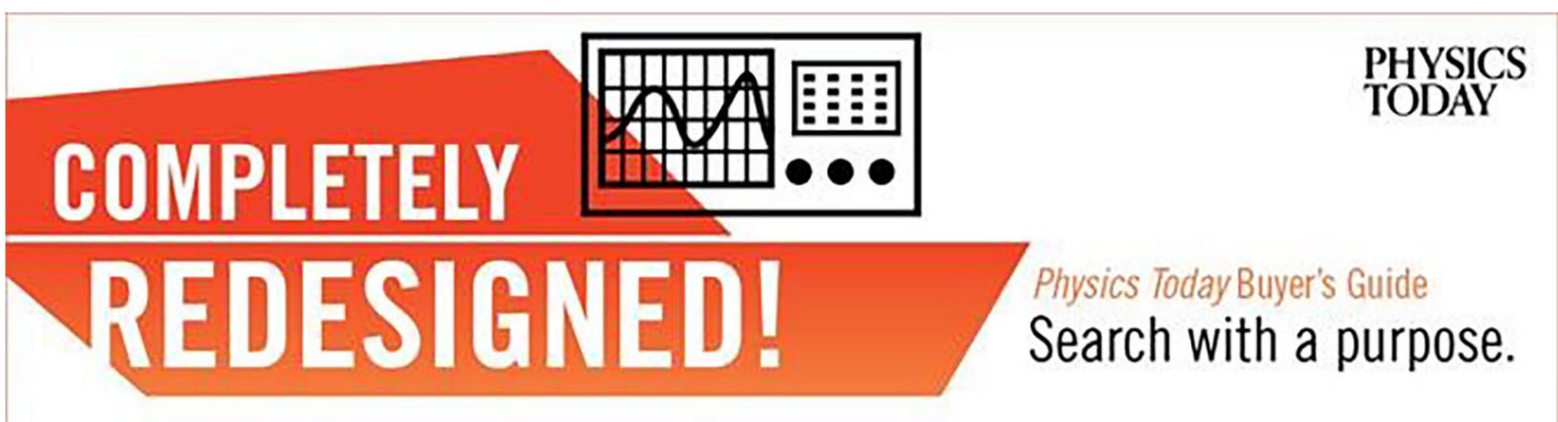




\title{
Properties of the water to boron nitride interaction: From zero to two dimensions with benchmark accuracy
}

\author{
Yasmine S. Al-Hamdani, ${ }^{1,2}$ Mariana Rossi, ${ }^{3}$ Dario Alfè,,$^{1,4}$ Theodoros Tsatsoulis, ${ }^{5}$ \\ Benjamin Ramberger, ${ }^{6}$ Jan Gerit Brandenburg, ${ }^{1,7,8}$ Andrea Zen, ${ }^{1,7}$ Georg Kresse, ${ }^{6}$ \\ Andreas Grüneis, ${ }^{5}$ Alexandre Tkatchenko, ${ }^{2}$ and Angelos Michaelides ${ }^{1,7}$ \\ ${ }^{1}$ Thomas Young Centre and London Centre for Nanotechnology, 17-19 Gordon Street, \\ London WC1H OAH, United Kingdom \\ ${ }^{2}$ Physics and Materials Science Research Unit, University of Luxembourg, L-1511 Luxembourg, Luxembourg \\ ${ }^{3}$ Fritz Haber Institute of the Max Planck Society, Faradayweg 4-6, 14195 Berlin, Germany \\ ${ }^{4}$ Department of Earth Sciences, University College London, Gower Street, London WC1E 6BT, United Kingdom \\ ${ }^{5}$ Max Planck Institute for Solid State Research, Heisenbergstraße 1, D-70569 Stuttgart, Germany \\ ${ }^{6}$ University of Vienna, Faculty of Physics and Center for Computational Materials Sciences, Sensengasse 8/12, \\ 1090 Wien, Austria \\ ${ }^{7}$ Department of Physics and Astronomy, University College London, Gower Street, \\ London WC1E 6BT, United Kingdom \\ ${ }^{8}$ Department of Chemistry, University College London, 20 Gordon Street, London WC1H 0AH, United Kingdom
}

(Received 29 May 2017; accepted 10 July 2017; published online 28 July 2017)

\begin{abstract}
Molecular adsorption on surfaces plays an important part in catalysis, corrosion, desalination, and various other processes that are relevant to industry and in nature. As a complement to experiments, accurate adsorption energies can be obtained using various sophisticated electronic structure methods that can now be applied to periodic systems. The adsorption energy of water on boron nitride substrates, going from zero to 2-dimensional periodicity, is particularly interesting as it calls for an accurate treatment of polarizable electrostatics and dispersion interactions, as well as posing a practical challenge to experiments and electronic structure methods. Here, we present reference adsorption energies, static polarizabilities, and dynamic polarizabilities, for water on BN substrates of varying size and dimension. Adsorption energies are computed with coupled cluster theory, fixed-node quantum Monte Carlo (FNQMC), the random phase approximation, and second order Møller-Plesset theory. These wavefunction based correlated methods are found to agree in molecular as well as periodic systems. The best estimate of the water/h-BN adsorption energy is $-107 \pm 7 \mathrm{meV}$ from FNQMC. In addition, the water adsorption energy on the $\mathrm{BN}$ substrates could be expected to grow monotonically with the size of the substrate due to increased dispersion interactions, but interestingly, this is not the case here. This peculiar finding is explained using the static polarizabilities and molecular dispersion coefficients of the systems, as computed from time-dependent density functional theory (DFT). Dynamic as well as static polarizabilities are found to be highly anisotropic in these systems. In addition, the many-body dispersion method in DFT emerges as a particularly useful estimation of finite size effects for other expensive, many-body wavefunction based methods. Published by AIP Publishing. [http://dx.doi.org/10.1063/1.4985878]
\end{abstract}

\section{INTRODUCTION}

Molecular adsorption on surfaces is a fundamentally important process in catalysis, gas storage, water purification, and many other areas. Water especially is ubiquitous, and in the absence of ultra-high vacuum (UHV), materials inevitably come into contact with it, which can have a substantial impact on surfaces both in industry and in nature. Even the gecko which is known for defeating gravity and climbing walls by adhesion from weak van der Waals (vdW) interactions ${ }^{1}$ has been found to lose its grip when its toepads become wet. ${ }^{2-4}$ Adsorption on the so-called vdW materials is particularly exciting as they exhibit peculiar long-range correlation interactions compared to bulk solids or molecules. ${ }^{5}$ In addition, recent efforts to treat unclean water have involved using low dimensional materials such as graphene and hexagonal boron nitride (h-BN) to adsorb harmful impurities. ${ }^{6,7} \mathrm{vdW}$ interactions also play an integral role in forming complex molecular crystal structures of compounds used in medicine. ${ }^{8}$ In order to understand and design new systems in this broad range of applications, an accurate knowledge of adsorption energies is useful and often essential.

Accurate adsorption energies on clean surfaces are difficult to obtain experimentally. That is partly because of the pristine surfaces under UHV that have to be prepared and, second, because few adsorption measurement techniques exist with the level of precision that is required to measure weak physisorption energies. Amongst those, single crystal adsorption calorimetry (SCAC) is a particularly elegant technique,,${ }^{9,10}$ but even with this, no adsorption energy for water on 2-dimensional (2D) surfaces has been reported yet. Fortunately, a few computational methods have emerged over the 
years that can be applied to periodic systems, as useful and reliable ways to calculate molecular adsorption energies on extended surfaces. These include the random phase approximation (RPA), diffusion Monte Carlo (DMC), and quantum chemical methods such as second order Møller-Plesset (MP2) theory and coupled cluster with single, double, and perturbative triple $[\operatorname{CCSD}(\mathrm{T})]$ excitations. These electronic structure methods have had numerous successes including, for example, the RPA prediction of the adsorption site for $\mathrm{CO}$ on metal surfaces ${ }^{11}$ where generalised gradient approximations (GGAs) within density functional theory (DFT) fail. Another example is the agreement between DMC and embedded coupled cluster theory for the surface energy ${ }^{12}$ and water adsorption energy ${ }^{13}$ on the $\mathrm{LiH}$ crystal-a material that spontaneously oxidises in moist air making any experimental measurement extremely difficult. Theoretically calculated reference adsorption energies are therefore helpful to experimentalists as well as to developers of computational methods.

A key challenge is having accurate electronic structure theories that we can solve for realistic extended surface models. However, thanks to the great improvements in codes and algorithms, surface adsorption problems have become increasingly accessible in recent years. ${ }^{11,14-28}$ Water adsorption on graphene is an exemplary case that has been computed with the RPA, DMC, ${ }^{14}$ and embedded $\operatorname{CCSD}(\mathrm{T}) .{ }^{15,27}$ However the reported adsorption energies range by up to $40 \%$, likely because the calculations involved a number of limitations and approximations which have not been quantified. Gauging the impact from different approximations is evidently desirable and would deliver greater understanding of the applicability of these methods.

In this study, we focus on the interaction of water with BN substrates that vary in size and dimension, from zero to 2D periodicity. In order to gain a better understanding about how the interaction varies, we compute reference adsorption energies, static polarizabilities, and dynamic polarizabilities for borazine (the $\mathrm{BN}$ analogue of benzene), boronene ${ }^{29}$ (BN analogue of coronene), and h-BN. These BN substrates are electronic insulators with band gaps exceeding $4 \mathrm{eV}$ at the GGA-DFT level, they contain lone pairs of electrons located on the nitrogen atoms, and they are geometrically analogous to carbon substrates. Intuitively, the interaction of molecular and surface BN substrates with water is expected to involve a mixture of polarizable electrostatics and dispersion interactions. This complex combination of interactions is widespread in biology and surface science, making the water/BN systems an excellent case study for establishing reference adsorption energies. Moreover, like graphene, the 2D h-BN surface also gives rise to long-range Coulomb interactions that are also relevant to adsorption on low-dimensional extended systems. ${ }^{30}$ However, unlike graphene and metallic surfaces, h-BN is an insulator, and therefore it is not well understood whether it gives rise to substantial non-additive dispersion interactions. Using the static and dynamic polarizabilities that are computed in this study, this important question on non-additive dispersion is addressed.

It is worth noting that a series of studies has ensued ${ }^{19,25,26}$ since we computed reference DMC interaction energy curves for water on h-BN. ${ }^{19}$ In our previous work, DMC interaction energy curves were computed for two stable configurations of water above the h-BN surface, and the adsorption energy was found to be $-85 \pm 5 \mathrm{meV}$ at the more favourable site, with one hydrogen atom of water pointing down towards a nitrogen atom in the h-BN surface. ${ }^{19}$ Furthermore, Wu et al. computed the interaction energy of water on a borazine molecule with DMC, MP2, and CCSD(T). ${ }^{25}$ Therein, the configuration is such that the two hydrogen atoms of water point down towards a boron and a nitrogen atom in borazine. ${ }^{25}$ Although the configuration that was used is unlikely to be the lowest in energy, they showed that $\operatorname{CCSD}(\mathrm{T})$ and $\mathrm{DMC}$ predicted the same interaction energy curves, with a maximum interaction of $\sim 75 \mathrm{meV}$ at $3.36 \AA$ separation. ${ }^{25}$ In addition, the RPA method has been applied to the water/h$\mathrm{BN}$ system with different unit cell sizes, ${ }^{26}$ estimating a finite size effect correction of $-16 \pm 8 \mathrm{meV}$. This correction arises because interactions in the water/h-BN system can extend beyond the finite unit cell employed, leading to spurious correlation interactions between electrons and their periodic images.

Here, we used DMC, lattice regularised DMC (LRDMC), $\operatorname{CCSD}(\mathrm{T}), \mathrm{RPA}$, and MP2 calculations to ascertain the best possible adsorption energy for water on the molecular and surface BN substrates. These benchmark calculations result in a particularly important finding: The water adsorption energy remains almost constant as the BN substrate size and dimension increase. To explain this peculiar finding and provide further benchmark data, we perform time-dependent DFT (TD-DFT) calculations of the static and dynamic polarizabilities for the water/BN systems. We find that there is considerable non-additivity in the dispersion interactions of these water/BN systems due to anisotropy in the polarizability. In what follows, the many-body dispersion (MBD) contribution $^{31,32}$ in DFT is found to agree with the RPA and TD-DFT, and importantly, it is shown to capture anisotropic interactions in the long-range limit in these systems. The results indicate that the MBD scheme is an efficient approach for estimating the long-range correlation interactions and can be used to determine the finite size effect correction for wavefunction based correlated electronic structure methods. Furthermore, a selection of DFT exchange-correlation $(x c)$ functionals is benchmarked for this set of water/BN substrates. Their performance varies, and we make note of which $x c$ functionals predict at least the same trend as the reference methods.

Details of the calculations and setups are given first in Sec. II followed by results in Sec. III. In Sec. III A, the interaction energy curves for a water monomer with borazine ( $\mathrm{BN}$ analogue of benzene, $\mathrm{B}_{3} \mathrm{~N}_{3} \mathrm{H}_{6}$ ), boronene ( $\mathrm{BN}$ analogue of coronene, $\mathrm{B}_{12} \mathrm{~N}_{12} \mathrm{H}_{12}$ ), and h-BN surface are reported, using a series of reference electronic structure methods along with previous results from DMC. In addition, the long-range correlation energy contribution to the water/hBN adsorption energy is determined with periodic MP2, RPA, and MBD, by computing increasing unit cell sizes of h-BN. This is followed by a brief assessment of some recently developed and otherwise widely used $x c$ functionals in Sec. III B. In Sec. III C, we present the static and dynamic 
polarizabilities of water/BN systems from TD-DFT and compare them with DFT+MBD. We close with conclusions in Sec. IV.

\section{METHODS}

In this study, the absolute interaction energies of water with BN substrates have been computed using DFT, RPA, MP2, CCSD(T), DMC, and LRDMC. The procedure for each of these methods is described shortly; however, let us first define the interaction energy and the systems being considered in Sec. II A.

\section{A. System setup}

The interaction energy between water and the substrate with all of the electronic structure methods considered here has been calculated in the same way as in our previous work, ${ }^{19}$

$$
E_{\text {int }}=E_{d}^{\text {tot }}-E_{\text {far }}^{\text {tot }}
$$

where $E_{d}^{\text {tot }}$ is the total energy of water and substrate at a given oxygen-substrate separation distance, $d$, and $E_{\text {far }}^{\text {tot }}$ is the total energy of water and substrate at $8 \AA$ oxygen-substrate distance. ${ }^{33}$ The water molecule has the same orientation on each substrate, with one hydrogen atom pointing down towards a nitrogen atom in the substrate. This makes for a cleaner comparison (see Fig. 1), and in this way, the same low energy configuration of water/h-BN is used as in Ref. 19. DFT geometry optimizations of the water/BN complexes confirmed that most stable orientation of the water molecule is the same on these three BN substrates. The lattice constant of h-BN used in all periodic calculations is $2.51 \AA$, in agreement with the experimental lattice constant. ${ }^{34}$ Geometries of the water/BN complexes in the minimum and far configurations can be found in the supplementary material. The interaction energy between water and substrate is plotted as a function of the perpendicular distance between the oxygen atom of the water molecule and the flat substrate. (a)

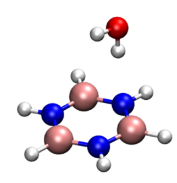

(c)

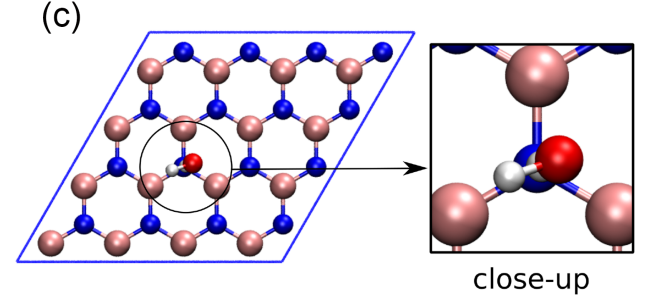

FIG. 1. (a) Water adsorbed on borazine $\left(\mathrm{B}_{3} \mathrm{~N}_{3} \mathrm{H}_{6}\right)$. (b) Water adsorbed on boronene $\left(\mathrm{B}_{12} \mathrm{~N}_{12} \mathrm{H}_{12}\right)$. (c) Water adsorbed on a $(4 \times 4)$ unit cell of a h-BN sheet with a close-up of the physisorption site. White spheres are hydrogen, red spheres are oxygen, pink spheres are boron, and blue spheres are nitrogen.

\section{B. DFT calculations}

The DFT calculations on the molecular BN systems have been conducted using the Vienna Ab initio Simulation Package (VASP.5.4.1) and standard projector augmented wave (PAW) potentials ${ }^{35,36}$ with a $500 \mathrm{eV}$ plane-wave cutoff energy and $\Gamma$-point sampling of reciprocal space. In VASP and Quantum Espresso, periodic boundary conditions (PBCs) must be set, and therefore for periodic calculations of the molecular water/borazine system, a $15 \times 15 \times 15 \AA^{3}$ unit cell was used. With this unit cell, the effect of a dipole correction is negligible $(<1 \mathrm{meV})$ in a Perdew-Burke-Ernzerhof (PBE) calculation with VASP. For the larger boronene molecule, a $20 \times 20 \times 16 \AA^{3}$ unit cell was used.

There are countless $x c$ functionals that could be assessed, and therefore, we have chosen only a few functionals in this study which are either new or widely used for such systems. Hence, a widely used GGA $x c$ functional, PBE, ${ }^{37}$ and a hybrid functional that contains a fraction of exact exchange, PBE0 ${ }^{38,39}$ are assessed. Given the importance of $\mathrm{vdW}$ interactions also, vdW-inclusive functionals are expected to be suitable in such systems, and therefore we also test optB86b-vdW ${ }^{40,41}$ and PBE+D3. ${ }^{42,43}$ In addition, we have tested the more recently developed strongly constrained and appropriately normalised (SCAN) functional of Sun et al. ${ }^{44}$ Furthermore, the interaction energy curves for water/BN substrates have been computed with $\mathrm{PBE}+\mathrm{MBD}^{31,32,37}$ using the all-electron FHI-AIMS code with tight settings. ${ }^{45}$

\section{Molecular quantum chemistry calculations}

The molecular systems were also calculated with quantum chemical codes (Gaussian, ${ }^{46}$ Molpro, ${ }^{47}$ and NWChem ${ }^{48}$ ) to obtain the MP2 interaction energy at $3.4 \AA$ water/substrate distance, with complete basis set (CBS) extrapolation ${ }^{49}$ using Dunning's aug-cc-pV(T,Q)Z basis sets. ${ }^{50,51}$ In order to compute interaction energies with reference accuracy, we use the linear scaling domain based pair natural orbital $\operatorname{CCSD}(\mathrm{T})$ [denoted here as the $\operatorname{LCCSD}(\mathrm{T})$ ] method $^{52}$ as implemented in the ORCA program package. ${ }^{53}$ The implementation has been optimized to use compact representations of all amplitudes and imposing block sparsity of tensors. ${ }^{54}$ The pair natural orbitals are used to employ the locality of electron correlation resulting in tunable thresholds to approach the full $\operatorname{CCSD}(\mathrm{T})$ "gold standard" of quantum chemistry. The domain and pair thresholds are used with the tight settings as necessary for the accurate computation of non-covalent interactions. Tightening the thresholds further for water/borazine led to a change in the interaction energy of less than $5 \mathrm{meV}$. The perturbative triples are computed with localized occupied orbitals and canonical virtual orbitals (sometimes abbreviated as T0). There is currently no evidence that a full canonical treatment yields better results. ${ }^{55}$ Indeed, the difference between our localized treatment and a full canonical $\operatorname{CCSD}(\mathrm{T})$ is small: estimated to be $4 \mathrm{meV}$ and $6 \mathrm{meV}$ for the CCSD and the triples contribution, respectively, for the water/boronene system in a cc-pVDZ basis set. Thus, the errors introduced by the truncation and non-canonical triples seem to be under control and below $10 \mathrm{meV}$ for the two systems we studied. 


\section{Periodic quantum chemistry calculations}

A developer version of VASP 5.4.2 was used for the periodic MP2 $2^{56}$ and RPA+SOSEX ${ }^{57-60}$ calculations for water on h-BN within the PAW framework. In all calculations, the electronic states of the $\mathrm{H}$ atoms were treated as valence states while the $1 s^{2}$ states of $\mathrm{B}, \mathrm{N}$, and $\mathrm{O}$ atoms were kept frozen. The $\Gamma$-point approximation was used to sample the first Brillouin zone. MP2 calculations utilize Hartree-Fock (HF) canonical orbitals, while the RPA calculations employ PBE orbitals. For the MP2 and RPA methods, the oneelectron states were expanded using a $600 \mathrm{eV}$ plane-wave energy cutoff. We employ a set of atom-centered functions to construct the unoccupied one-electron states, mapped onto a plane-wave representation. Dunning's contracted aug-ccpVDZ (AVDZ) and aug-cc-pVTZ (AVTZ) basis sets ${ }^{50,51}$ were used as atom-centered functions. With the rediagonalization of the Fock matrix in this newly constructed virtual space, we mimic AVDZ and AVTZ basis sets in a plane-wave basis suitable for periodic correlated calculations. ${ }^{61}$ Counterpoise corrections (CP) to the basis set superposition error (BSSE) were included in all correlated quantum-chemical calculations which involve pseudized Gaussian type orbitals (PGTOs). If not stated otherwise, we extrapolate the correlation energies to the complete basis limit using $\mathrm{AV}(\mathrm{D}, \mathrm{T}) \mathrm{Z}$ results. The twoelectron integrals are calculated using an auxiliary plane-wave basis in VASP. ${ }^{56,62,63}$ The kinetic energy cutoff $E_{\chi}$ such that $\frac{\hbar^{2} \mathbf{G}^{2}}{2 m_{e}}<E_{\chi}$ defining this auxiliary plane-wave basis set was set to $300 \mathrm{eV}$. All reported MP2 and RPA adsorption energies have been checked for convergence with respect to this cutoff. We note that the canonical implementation of MP2 in VASP scales as $\mathcal{O}\left(N^{5}\right)^{56}$ although recently an implementation scaling as $O\left(N^{4}\right)$ became available as well. ${ }^{64}$ The RPA calculations with PGTOs were also performed in an $\mathcal{O}\left(N^{5}\right)$ implementation in VASP allowing for the inclusion of secondorder screened exchange (RPA+SOSEX) for a more accurate description. ${ }^{57}$

Additional RPA calculations were preformed using a cubic scaling implementation in VASP ${ }^{58-60}$ and the full planewave basis in contrast to the PGTOs. PBE orbitals were used ${ }^{65}$ along with a $430 \mathrm{eV}$ plane-wave energy cutoff and the $\Gamma$-point approximation to sample the first Brillouin zone. In this case, the cutoff for the response functions was set to the default value of $287 \mathrm{eV}$. The results were extrapolated to the infinite basis set limit using the internal VASP extrapolation. This assumes that errors drop off like one over the basis set size. ${ }^{66,67}$ A quadrature with 8 grid points was used for the evaluation of the imaginary time and frequency integrations. In addition, the contribution from GW single excitations (GWSEs) was computed based on the work of Klimeš et al. ${ }^{60}$ We stress that adsorption energies calculated within the RPA using the cubic scaling implementation and the $\mathcal{O}\left(N^{5}\right)$ implementation with PGTOs are in good agreement. ${ }^{68}$

\section{E. Fixed-node quantum Monte Carlo (FNQMC)}

Herein we report results coming from two different fixednode quantum Monte Carlo (FNQMC) approaches: standard DMC $^{69}$ and LRDMC. ${ }^{70,71}$ These are both projection Monte Carlo methods: they can access the electronic ground state energy of the system by iteratively projecting an initial trial wave function $\psi_{T}$ onto the ground state, with the constraint that the projected wave function $\Phi$ has the same nodal surface of an appropriately chosen guiding function $\psi_{G}$ (fixed node approximation). ${ }^{69,72}$ Typically, $\psi_{T}=\psi_{G}=\psi_{\mathrm{VMC}}$, where $\psi_{\mathrm{VMC}}$ is the best function obtained within a variational Monte Carlo approach. Whenever $\psi_{G}$ has the exact nodal surface, the approach is exact, otherwise it gives the best approximation of the ground state given the fixed node constraint.

In projection Monte Carlo approaches, there is a second approximation in how the projection is performed, and it is different in DMC and LRDMC. The projection in DMC comes from the imaginary time Schrödinger equation; it is implemented as an imaginary time evolution, where a time step $\tau$ has to be chosen. The chosen $\tau$ is a trade-off between accuracy and computational cost: the latter is $\propto 1 / \tau$, but the projection is exact only in the limit of $\tau \rightarrow 0$. Recently, an improved DMC algorithm ${ }^{73}$ that solves a size-inconsistency issue at finite values of $\tau$ was introduced, and it was shown that it dramatically reduces the time step errors in the evaluation of interaction energies.

Here, DMC calculations were performed with the CASINO code ${ }^{74}$ using Slater-Jastrow type trial wavefunctions in which the Jastrow factor contains electron-nucleus, electron-electron, and electron-electron-nucleus terms. We used Trail and Needs pseudopotentials $\mathrm{s}^{75,76}$ for all atoms. The DMC procedure is similar to that used in Refs. 19 and 77. The initial single particle wavefunctions for use in DMC were obtained from DFT plane-wave calculations using Quantum Espresso v.5.0.3. ${ }^{78}$ A standard $4082 \mathrm{eV}$ (300 Ry) energy cutoff was applied, and for efficiency, the resulting wavefunctions were expanded in terms of B-splines ${ }^{79}$ using a grid multiplicity of 2.0 corresponding to a grid spacing, $a=\pi / 2 G_{\max }$, where $G_{\max }$ is the plane wave cutoff wavevector. Trial wavefunctions were generated using the local-density approximation $(\mathrm{LDA})^{80}$ which has been validated for weak interactions in previous work. ${ }^{14}$ Variational Monte Carlo (VMC) was used to optimize the Slater-Jastrow type trial wavefunctions. In the evaluation of weak interactions in systems such as those under consideration in this work, the standard Jastrow correlated single Slater determinant (Slater-Jastrow) has proved to be sufficiently accurate, as established in a number of studies. ${ }^{19,28,77,81-91}$

In DMC, the locality approximation was utilized ${ }^{92}$ with a time step, $\tau_{\text {standard }}$, of 0.015 a.u. which was tested against a time step, $\tau_{\text {small }}$, of 0.005 a.u for the water/borazine interaction. The interaction energy agrees within the error bars of less than $5 \mathrm{meV}$ for $\tau_{\text {standard }}$ and $\tau_{\text {small }}{ }^{93}$

LRDMC, on the other hand, is based on the spatial discretization of the molecular Hamiltonian on a lattice of mesh size $a$, and it resorts to the projection scheme used also in the Green function Monte Carlo algorithm. ${ }^{94,95}$ The error induced by the finite mesh size $a$ is analogous to the time step error appearing in standard DMC calculations; it can be controlled by performing several calculations with different values of the mesh $a$ and finally extrapolating to the continuum limit $a \rightarrow 0$, but in practical cases, it is sufficient to consider the results for a mesh $a$ small enough that the expected finite-mesh bias is negligible. ${ }^{96}$ 
The LRDMC results reported in this work have been obtained with the TurboRVB package developed by Sorella and co-workers. ${ }^{97}$ We used a Slater-Jastrow trial wavefunction, but the setup for the LRDMC calculations slightly differs from that of the DMC calculations as a consequence of the different implementations of the algorithms in TurboRVB and CASINO.

For a more detailed description of the functional form of the wavefunction implemented in TurboRVB, see Ref. 98. Core electrons of $\mathrm{B}, \mathrm{N}$, and $\mathrm{O}$ atoms ${ }^{99}$ have been described via scalar-relativistic energy-consistent Hartree-Fock (HF) pseudopotentials of Burkatzki et al. ${ }^{100,125}$ The coefficients of the molecular orbitals have then been optimized by performing an LDA calculation, using the DFT code included in the TurboRVB package. ${ }^{101}$ The Jastrow factor used here consists of terms that account for the electron-electron, electron-nucleus, and electron-electron-nucleus interactions. ${ }^{102}$ The exponents of the Jastrow atomic orbitals have been fixed to the values obtained from the optimisation in the water/borazine system. All the other parameters of the Jastrow factor have been optimized for each specific configuration. In the LRDMC, we used a mesh $a$ of 0.3 a.u. We verified in the water/borazine system that we have no bias given by the choice of the pseudopotentials (indeed, by using the Trail and Needs pseudopotentials, ${ }^{75,76}$ we obtained results in agreement with those from the work of Burkatzki et al., within the statistical error margin) or by the choice of the mesh $a$. This is not a surprise since the approach here adopted was already tested in a number of other systems. ${ }^{28,90,98,103-105}$

Energies from quantum Monte Carlo approaches-both DMC and LRDMC - are affected by finite size simulation errors, ${ }^{106,107}$ which in the h-BN system under consideration here is mostly arising from the long-range Coulomb interactions. There are currently three methods available to deal with them: the use of the model periodic Coulomb (MPC) potential for the long-range interaction, ${ }^{108-110}$ a posteriori corrections to the energy as prescribed by Chiesa et al., ${ }^{111}$ or Kwee, Zhang, and Krakauer (KZK). ${ }^{112}$ In this work, we have used MPC, one of the most accurate approaches. ${ }^{113}$

In all systems, the interaction energy was evaluated as the difference between the bound configuration and the configuration with the water molecule far away, according to Eq. (1). This choice leads to an efficient cancellation of errors due to the finite time step (for DMC) ${ }^{73}$ or mesh (for LRDMC) and finitesize simulation. ${ }^{28}$ For the water/borazine and water/boronene systems, we used open boundary conditions in FNQMC calculations. For the water/h-BN system, we used 3D periodic boundary conditions, having set the distance between h-BN sheets to $16 \AA$.

\section{RESULTS}

Reference interaction energy curves for water on borazine, boronene, and h-BN at the most favourable adsorption site have been computed, contributing to the body of knowledge that has been reported previously. ${ }^{19,25}$ The long-range correction to the adsorption energy in the water/h-BN system is determined from FNQMC, RPA, MP2, and MBD at the most favourable adsorption site. In what follows, it will be shown that excellent agreement is achieved amongst the benchmark methods, and we provide our best prediction of the water $/ \mathrm{h}$ $\mathrm{BN}$ adsorption energy. We also compare some $x c$ functionals and find that their performance varies across the dimensions. Later, we present TD-DFT results of the static and dynamic polarizabilities for these water/BN systems, and using these properties, we explain the trend observed for the water adsorption energy on $\mathrm{BN}$ substrates from zero to $2 \mathrm{D}$. In doing so, we also demonstrate the accuracy of the MBD method in DFT.

\section{A. Molecular adsorption energies on BN substrates from zero to 2D}

Reference DMC interaction energy curves have been computed here for water on borazine and boronene and are shown in Fig. 2. We are referring to the $\mathrm{BN}$ analogue of coronene as boronene here, also used by $\mathrm{Wu}$ et al. in an extrapolation scheme to predict the interaction energy of water on h-BN. ${ }^{25}$ In addition, Al-Hamdani et al. computed the interaction energy curve for water at boron and nitrogen sites in h-BN from DMC (the latter is also shown in Fig. 2). Later, Wu et al. performed direct RPA calculations and estimated a $16 \pm 8 \mathrm{meV}$ finite size error (FSE) correction. ${ }^{26}$ The finite size correction is necessary because of the long-range Coulomb interactions between fluctuating charges that are exhibited by this system. Here, we first demonstrate the effect of single excitations (SEs) and second order screened exchange (SOSEX) on the RPA interaction energies, and second, we find the extent of the long-range charge fluctuations and therefore the finite size correction with periodic MP2, RPA, and the MBD method in DFT. In addition, we also compute the FSE in FNQMC interaction energies from the KZK and MPC methods.

The DMC interaction energy curves for water/borazine and water/boronene have been computed here using the configurations of water shown in Fig. 1 and a DMC time step of 0.015 a.u. For water/borazine, the DMC interaction energy minimum appears at $3.32 \AA$ in Fig. 2(a). Further reference interaction energies from LCCSD(T), LRDMC, DMC (with 0.005 a.u. time step), MP2, and RPA are reported for water/borazine at this $3.32 \AA$ oxygen-ring distance in Table I. The LRDMC, $\operatorname{LCCSD}(\mathrm{T})$, and MP2 results agree with the DMC interaction energy within the stochastic error bars that are $<5 \mathrm{meV}{ }^{114}$ Note that we have also computed the conventional CCSD(T) interaction energy for the water/borazine system, and at $-119 \mathrm{meV}$, it is in close agreement with LCCSD(T). The direct RPA is instead underestimating the water/borazine interaction by $\sim 20 \mathrm{meV}$. Indeed, the direct RPA has been found to underbind weakly interacting systems in general. ${ }^{115}$ This underestimation can be alleviated by including GWSE correction, and for water/borazine, this leads to an $20 \mathrm{meV}$ improvement, bringing the RPA+GWSE into agreement with MP2, DMC, LRDMC, and LCCSD(T). The GWSE contribution is determined by calculating the first order difference of the density matrix between DFT and the GW approximation and correspondingly, a first order correction to the HF energy functional. ${ }^{60}$ This approach yields a slightly smaller correction than the single excitation correction of Ren and coworkers. ${ }^{115}$ In the GWSE approximation, correlation effects in the density matrix are more accurately included. In the 

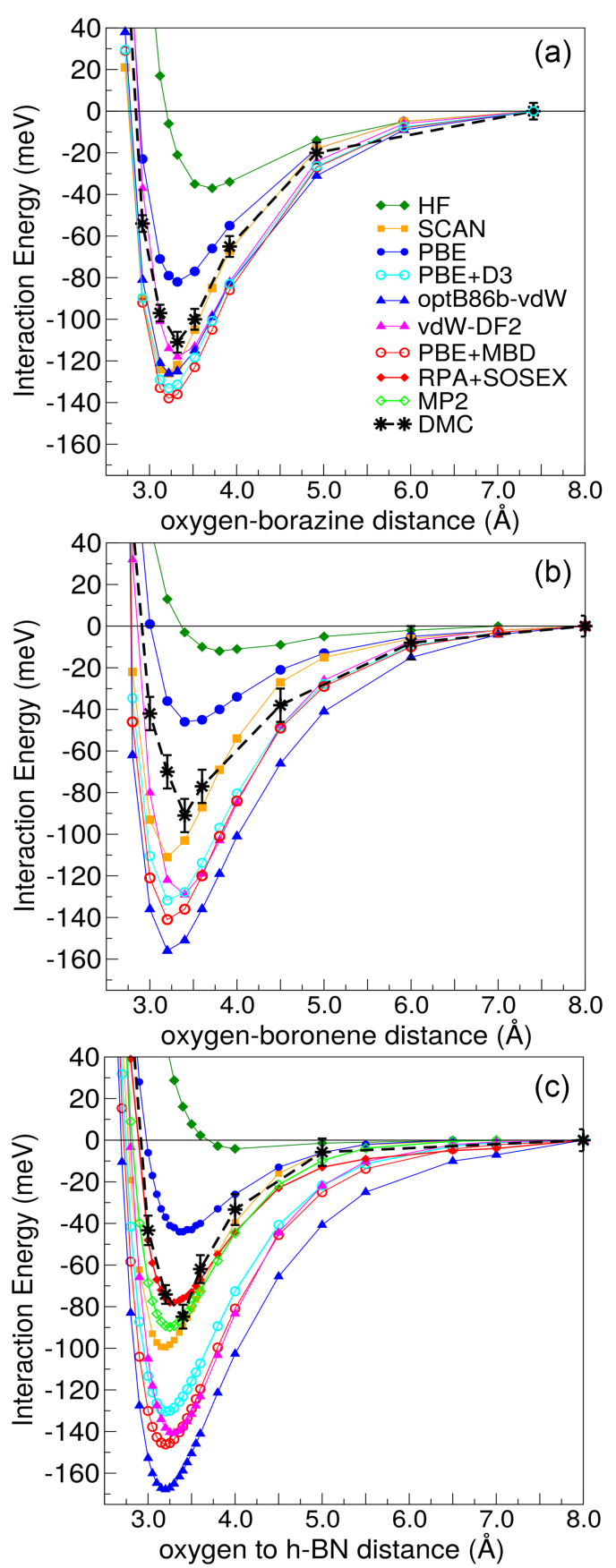

FIG. 2. Interaction energy curves for water situated above the $\mathrm{N}$ site in the (a) borazine molecule $\left(\mathrm{B}_{3} \mathrm{~N}_{3} \mathrm{H}_{6}\right)$, (b) boronene molecule $\left(\mathrm{B}_{12} \mathrm{~N}_{12} \mathrm{H}_{12}\right)$, and (c) h-BN surface. The DMC data points for water/h-BN are taken from a previous study, see Ref. 19. The DMC data shown have been computed with $\tau_{\text {standard }}=0.015$ a.u. The data points are connected by lines simply to guide the eye.

present case, the standard SE contributions are about $25 \mathrm{meV}$, whereas the GWSE contributions amount to the already quoted $20 \mathrm{meV}$.

On the larger boronene molecule, the water interaction minimum appears at $3.40 \AA$ on the DMC interaction energy curve shown in Fig. 2(b). The main difference between boronene and borazine is their size, and hence, the water adsorption energy is naively expected to be larger on boronene than on the smaller borazine molecule due to dispersion. However, using the $3.40 \AA$ A oxygen-boronene separation distance in
TABLE I. Interaction energies using different methods for water with borazine (at $3.32 \AA$ oxygen height above ring), boronene (at $3.40 \AA$ oxygen height from the molecule), and h-BN. Where possible, the oxygen/h-BN distance at the minimum of the interaction energy curve with the corresponding method is shown in parentheses in $\AA$. Canonical coupled cluster theory has been used for water/borazine whilst local coupled cluster theory has been used for water/boronene. The water/h-BN adsorption energies include a finite size correction of $20 \mathrm{meV}$, except for the FNQMC results which are corrected with the MPC method $(10 \mathrm{meV})$. Converged DFT results are reported in the bottom panel.

\begin{tabular}{lccc}
\hline \hline Method & Water/borazine & Water/boronene & Water/h-BN \\
\hline (L)CCSD(T)/CBS & -123 & -109 & $\mathrm{n} / \mathrm{a}$ \\
LRDMC & $-122 \pm 4$ & $-107 \pm 5$ & $-107 \pm 7(3.40)$ \\
DMC & $-117 \pm 3$ & $-96 \pm 8$ & $-95 \pm 5(3.40)$ \\
MP2/CBS & -120 & -113 & $-110(3.25)$ \\
(L)CCSD/CBS & -102 & -90 & $\mathrm{n} / \mathrm{a}$ \\
RPA+SOSEX+GWSE & $\mathrm{n} / \mathrm{a}$ & $\mathrm{n} / \mathrm{a}$ & $-113(3.25)$ \\
RPA+GWSE & -112 & $\mathrm{n} / \mathrm{a}$ & $-108(3.25)$ \\
RPA & -92 & $\mathrm{n} / \mathrm{a}$ & $-89(3.36)$ \\
\hline PBE & -82 & -46 & $-44(3.40)$ \\
SCAN & -122 & -103 & $-99(3.20)$ \\
PBE+D3 & -131 & -132 & $-136(3.20)$ \\
PBE+MBD & -138 & -141 & $-146(3.25)$ \\
optB86b-vdW & -125 & -151 & $-168(3.20)$ \\
vdW-DF2 & -118 & -129 & $-141(3.30)$ \\
\hline \hline
\end{tabular}

Table I, the water/boronene $\operatorname{LCCSD}(\mathrm{T})$ interaction energy is $-109 \mathrm{meV}$ which is $\sim 10 \mathrm{meV}$ less than the water/borazine interaction. MP2 is also in close agreement with $\operatorname{LCCSD}(\mathrm{T})$ for water/boronene. However, the LCCSD results show that the contribution from perturbative triple excitations is a considerable $20 \mathrm{meV}$ for the water/borazine and water/boronene systems. Therefore, the performance of MP2 appears to be fortuitous. This has important implications for extrapolation schemes, where it is not given that the fortuitous behavior in MP2 is systematic. As seen for water/borazine, LRDMC agrees with $\operatorname{LCCSD}(\mathrm{T})$, predicting an interaction energy of $-107 \pm 5 \mathrm{meV}$ for water/boronene. We also see an indication that DMC predicts a slightly smaller interaction energy than LCCSD(T) and LRDMC. The difference is small considering the stochastic error on the DMC energy, but given the slightly better agreement between LRDMC and LCCSD(T), we suggest that LRDMC provides the best prediction for water adsorption on $\mathrm{h}-\mathrm{BN}$ in the following.

Interaction energy curves are also shown for water/h-BN from DMC, RPA, RPA+SOSEX, and MP2 [see Fig. 2(c)]. MP2 calculations are performed for the periodic system, with the same unit cell as for the other methods. The RPA, RPA+SOSEX, and MP2 energies have been extrapolated to the complete basis set limit using pseudized aug-cc$\mathrm{pV}(\mathrm{D}, \mathrm{T}) \mathrm{Z}$ basis sets. Note that the interaction energy curves in Fig. 2(c) are computed for the $(4 \times 4)$ unit cell of h$\mathrm{BN}$, and the values reported now are taken directly from these curves without including a correction for finite size effects. It can be seen from Fig. 2(c) that the RPA interaction energies agree within the stochastic error of DMC interaction energies-in line with the findings of Wu et al. ${ }^{26}$ The interaction energy of water according to the RPA is $-69 \mathrm{meV}$ at the minimum (3.36 $\AA$ ). From Fig. 2, the SOSEX 
correction to direct RPA increases the interaction energy on the order of $\sim 5 \mathrm{meV}$, especially when water is close to the h-BN surface at $<3.5 \AA$ height. Therefore, the RPA+SOSEX interaction energy of water on $\mathrm{h}-\mathrm{BN}$ is $-74 \mathrm{meV}$ at the minimum $(3.25 \AA)$ on the RPA+SOSEX interaction energy curve. Earlier, however, GWSE was found to contribute $20 \mathrm{meV}$ towards the interaction energy of water/borazine, and similarly here, GWSE contributes $20 \mathrm{meV}$ to the interaction energy of water on h-BN at the minimum. As such, the $\mathrm{RPA}+\mathrm{SOSEX}+\mathrm{GWSE}$ adsorption energy of water/h-BN is $-94 \mathrm{meV}$. Meanwhile, MP2 predicts an adsorption energy of $-88 \mathrm{meV}$ for water/h-BN, also at $3.25 \AA$. It should be noted that MP2 theory does not contain singles contributions because of Brillouin's theorem. The interaction energies from RPA+SOSEX+GWSE and MP2 are slightly larger than the DMC interaction energy although these are still within a few $\mathrm{meV}$ of the DMC adsorption energy $(-85 \pm 5 \mathrm{meV})$. Furthermore, the LRDMC interaction energy of water on h-BN at $3.40 \AA$ is $-97 \pm 7 \mathrm{meV}$. Thus, all of the aforementioned reference methods are demonstrably in excellent agreement. However, as previously indicated by Wu et al., ${ }^{26}$ there is a long-range Coulomb interaction that arises in methods that account for fluctuating dipoles which is not fully captured in the $(4 \times 4)$ unit cell of $\mathrm{h}-\mathrm{BN}$ at the $\Gamma$-point. Since this interaction extends beyond the $(4 \times 4)$ unit cell of h-BN, it requires a great deal of computational effort to capture with expensive many-body wavefunction based methods. As such, the agreement between different methods has scarcely been verified on an extended surface. Let us address this particular point in the following.

Long-range Coulomb type interactions from the fluctuation of electrons, otherwise referred to as long-range correlation energy, can extend to the nanometre scale ${ }^{30}$ and are expected to be particularly important in low dimensional systems. In methods that account for charge fluctuations (and therefore $\mathrm{vdW}$ interactions) explicitly, whether it is by $s \rightarrow p$ excitations for coupled harmonic oscillators ${ }^{116}$ or via more general excitation mechanisms in the RPA and MP2 theory, the fluctuations can give rise to spurious interactions with their periodic images. As such, correlated methods are affected by FSE any time they are providing the energy of a macroscopic system by employing PBC. Since the interaction energy is evaluated as the difference between two periodic systems here, a big, but only partial, cancellation of the FSE can be expected. These FSEs are much larger than those observed in (effectively) independent electron methods such as HF and DFT.

To establish the extent of FSE in methods that account for correlation effects explicitly, MP2, ${ }^{117}$ RPA, RPA+GWSE, and $\mathrm{PBE}+\mathrm{MBD}$ interaction energies for water/h-BN have been computed in increasing supercell sizes. More specifically, h-BN systems with as many as 98 surface atoms in the unit cell have been computed with MP2 and RPA in order to establish the long-range behavior of the water interaction energy. MP2 calculations have been performed using a pseudized AVTZ basis set, whereas RPA calculations utilize the cubic scaling implementation in VASP. Note that this is a factor of 2 larger than the typical unit cells used in molecular adsorption studies on low dimensional surfaces. ${ }^{15,19,23-25}$
TABLE II. Interaction energies in meV for water on h-BN at $3.25 \AA$ calculated using $\Gamma$-point sampling only with increasing supercell sizes. $\mathrm{N}$ is the number of atoms in the h-BN substrate unit cell. $\Delta E_{\text {int }}^{f s e}$ is the difference in the interaction energy due to long-range Coulomb interactions for water with 32 and 98 atoms in the h-BN unit cell.

\begin{tabular}{lcccc}
\hline \hline $\mathrm{N}$ & MP2 & RPA & RPA+GWSE & PBE+MBD \\
\hline $18(3 \times 3)$ & -70 & -34 & -58 & -88 \\
$32(4 \times 4)$ & -93 & -64 & -84 & -125 \\
$50(5 \times 5)$ & -106 & -76 & -95 & -139 \\
$72(6 \times 6)$ & -113 & -78 & -97 & -145 \\
$98(7 \times 7)$ & -116 & -84 & -103 & -148 \\
\hline$\Delta E_{\text {int }}^{f s e}$ & -23 & -20 & -19 & -23 \\
\hline \hline
\end{tabular}

This is a brute force approach, computationally very demanding because the scaling with size of the correlated methods, and as such, the FSE is computed only near the minimum of the interaction energy curve. The results are reported in Table II and shown in Fig. 3(a). The finite size effect correction to the interaction energy of water in a $(4 \times 4)$ unit cell of h-BN is denoted here as $\Delta E_{\text {int }}^{f s e}$. Importantly, the various reference methods we have used, namely, MP2, RPA, and RPA+GWSE, as well as the MBD method in DFT, all estimate $\Delta E_{\text {int }}^{f s e} \approx 20 \mathrm{meV}$ (see Table II) when $\Gamma$-point calculations are performed. In addition to establishing the agreement amongst these methods, this $20 \mathrm{meV}$ is approximately $25 \%$ of the reference adsorption energy computed in a $(4 \times 4)$ unit cell of $\mathrm{h}-\mathrm{BN}$ and is therefore a considerable contribution. It is worth noting in Fig. 3(a) the different convergence of DFT calculations with the PBE functional, which represents a typical case for a method with effectively independent electrons. The PBE interaction energy of water $/ \mathrm{h}-\mathrm{BN}$ is almost converged in a $(3 \times 3)$ unit cell of $\mathrm{h}-\mathrm{BN}$. What about the convergence of vdW-DFs or dispersion corrections such as the D3? Figure 3(a) shows that in these methods, the convergence is similar to that of PBE, i.e., the dispersion interaction given by these methods is fully captured in the $(4 \times 4)$ unit cell of h-BN. This behavior is expected since vdW-DFs and dispersion corrections evaluate dispersion interactions indirectly, from the density of electrons in the former and from the environment dependent isotropic atomic $\mathrm{C}_{6}$ dispersion coefficients in the latter-and not explicitly as in the RPA, MP2, or MBD. Furthermore, in the D3 method, the interactions are not only calculated in a minimum image convention, but the long-range interactions are summed over repeated images up to very large distances that exceed the actual simulation cell.

The results shown in Fig. 3(a) signify that sampling at the $\Gamma$-point of the $(3 \times 3)$ or $(4 \times 4)$ supercell of h-BN evidently does not describe the long-range correlation effects very accurately within the wavefunction based correlated methods. However, the MBD method converges in the same manner as the RPA and MP2 with supercell size when using the $\Gamma$-point only. Therefore, the MBD method can be used as an effective and efficient estimate of the FSE for other more expensive, wavefunction based correlated methods. On the other hand, a faster cell size convergence in the wavefunction based correlated methods can be obtained by improving the k-point 

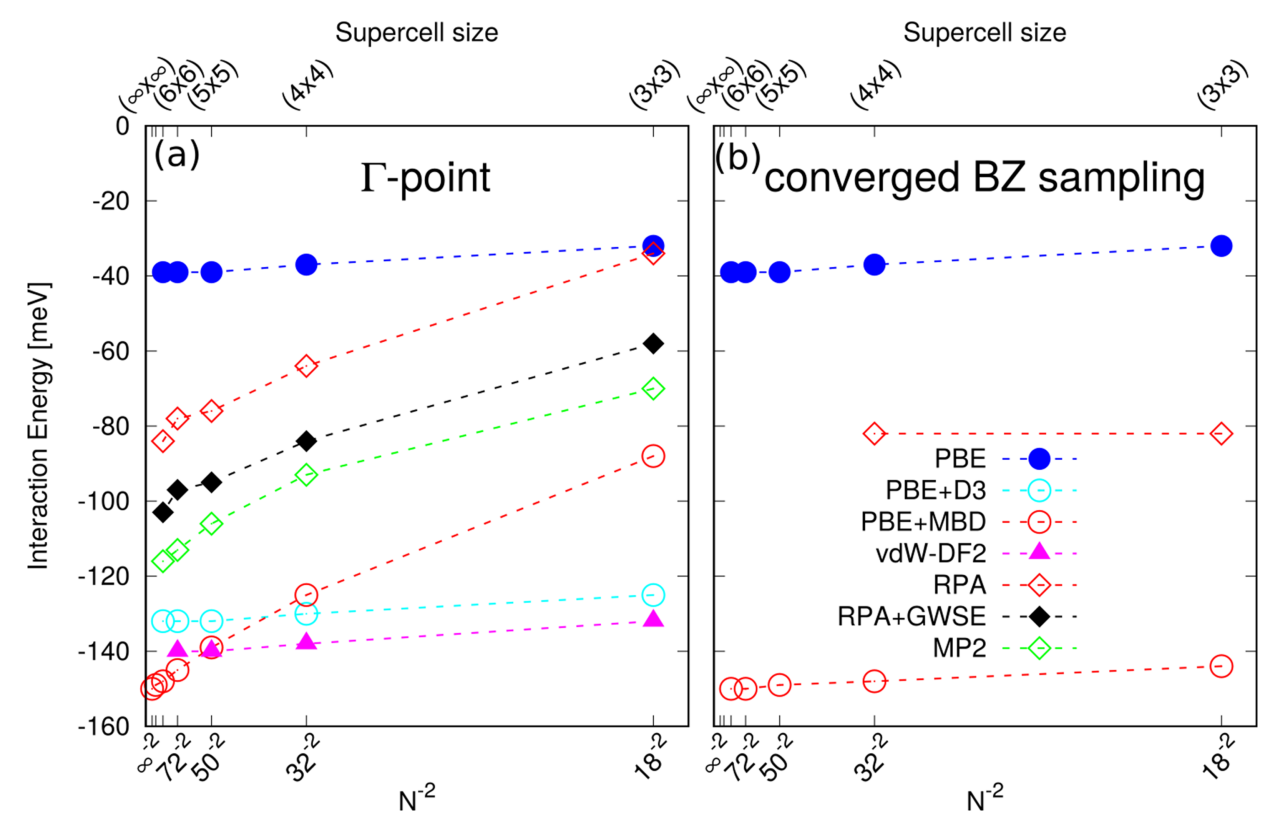

FIG. 3. Interaction energy of water/h$\mathrm{BN}$ at $3.25 \AA$ height for different supercells with $\mathrm{N}$ being the number of atoms in the h-BN unit cell. (a) Interaction energies with $\Gamma$ point sampling only. (b) Interaction energies with converged k-point sampling of the Brillouin zone (BZ). The $\mathrm{x}$-axis is scaled as $1 / \mathrm{N}^{2}$ which is the expected scaling from pairwise additive interaction. The interaction energies are shown for RPA, RPA+GWSE, MP2, PBE+MBD, PBE, $\mathrm{PBE}+\mathrm{D} 3$, and vdW-DF2. sampling. For instance, the adsorption energy for the $(3 \times 3)$ cell calculated with RPA sampling the Brillouin zone with a $4 \times 4 \times 1$ grid is $-82 \mathrm{meV}$, which is much closer to the low coverage value of $-84 \mathrm{meV}$ than the value of $-34 \mathrm{meV}$ obtained for a $(3 \times 3)$ cell at $\Gamma$, as reported in Table II. Similarly, in the $(4 \times 4)$ cell, the RPA value obtained by using the $\Gamma$ and the $\mathrm{K}$ points in the Brillouin zone yields a value for the adsorption energy of $-82 \mathrm{meV}$ instead of $-64 \mathrm{meV}$ for the $\Gamma$-point. Likewise, most implementations of the MBD method include very long-range dipole-dipole interactions by either calculating the MBD interactions in a supercell of the actually used cell or by explicitly using a denser k-point sampling than that which is used in the underlying DFT electronic structure calculations. Figure 3(b) shows the adsorption energy values for RPA, PBE, and PBE+MBD, obtained for different supercells for a converged $\mathbf{k}$-point sampling of the Brillouin zone. In most cases, the $1 / \mathrm{N}^{2}$ convergence observed in Fig. 3(a) can be overcome resulting in a converged behavior as shown in Fig. 3(b).

Fortunately, much cheaper methods for the evaluation of the FSE in correlated methods exist, and they are only slightly less accurate than the brute force method. In the FNQMC community, there are several schemes such as the KZK, MPC, and Chiesa methods. We have computed the MPC correction in FNQMC and found it to be $10 \mathrm{meV}$. Since this correction is based on the FNQMC calculations, we use this value to correct both the LRDMC and DMC adsorption energies of water on $\mathrm{h}-\mathrm{BN}$ reported in Table I. The DFT based KZK correction to FNQMC has also been computed out of interest and it results in $\Delta E_{\text {int }}^{f s e}=17 \mathrm{meV}$. Although the KZK method is not as accurate as the MPC method, it predicts a reasonable estimate of the FSE given that it is less computationally demanding. Thus the best estimate of the water/h-BN adsorption energy with the MPC correction from DMC is $-94 \pm 5 \mathrm{meV}$ and $-107 \pm 7 \mathrm{meV}$ from LRDMC. Recently, corrections for the correlated quantum chemistry methods have also been introduced, ${ }^{118}$ and such schemes yield approximately a $20 \mathrm{meV}$ correction for water adsorption on a $(4 \times 4)$ unit cell of h-BN for MP2 and RPA. ${ }^{119}$
To briefly summarise the results of this section, water/BN interaction energies have been reported from a wide range of electronic structure methods. Given that methods like LCCSD(T), LRDMC, DMC, MP2, and RPA are often regarded as reference methods, it is important to demonstrate that these different electronic structure theories predict the same interaction energies for realistic surface models. With a great deal of computational effort that has been expended here, agreement between the aforementioned wavefunction based correlated methods has been demonstrated for water adsorption on molecular and extended BN substrates. All of these electronic structure methods involve practical approximations that have had to be carefully addressed in order to predict accurate adsorption energies. The series of calculations in this study lead to an improved prediction of the water/h-BN adsorption energy, that is, $-107 \pm 7 \mathrm{meV}$ with LRDMC. This carefully established water adsorption energy on h-BN presents a challenge for experiments, and hence it would be particularly exciting to see future experimental adsorption studies focusing on this system. In addition, the reference information in this study is intended to help the development of computational methods, and in Sec. III B, we benchmark a selection of $x c$ functionals from DFT.

\section{B. Benchmarking $x c$ functionals in DFT}

Let us focus now on the performance of DFT $x c$ functionals. Figure 2 shows the interaction energy curves of water on the BN substrates from PBE, PBE+D3, PBE+MBD, optB86b$\mathrm{vdW}$, and SCAN $x c$ functionals for comparison. We consider two important aspects when assessing these $x c$ functionals. First, we compare the absolute adsorption energy of water on each substrate to the reference methods. More specifically, for the water/h-BN system, we compare to the long-range corrected adsorption energies in Table I. Second, we consider the relative trend of the water adsorption energy from the small molecule to the extended BN surface, keeping in mind that LRDMC and other reference methods predict that the 
interaction of water is $10-15 \mathrm{meV}$ less on boronene and h-BN compared to borazine.

Starting with the most widely used $x c$ functional, PBE underestimates the interaction energy in both the molecular systems and on the extended h-BN surface by as much as $50 \%$. This is expected since with this functional, vdW interactions are not treated. Note that the hybrid functional PBE0 is not shown in Fig. 2 because it was found to overlap with PBE in the water/borazine and water/boronene systems, as well as being within $10 \mathrm{meV}$ of PBE in the water/h-BN system. Despite severely underestimating the adsorption energies, PBE and PBE0 correctly predict that the water/boronene interaction energy is less than the water/borazine interaction energy.

Dispersion inclusive $x c$ functionals such as optB86b-vdW and vdW-DF2 are generally considered as appropriate methods for predicting the properties of layered materials and $\mathrm{vdW}$ dominated complexes. From Fig. 2, it can be seen that optB86b-vdW and vdW-DF2 provide good agreement with reference interaction energies for water/borazine near the minimum at $3.32 \AA$, predicting interaction energies of $-126 \mathrm{meV}$ and $-118 \mathrm{meV}$, respectively. However, these dispersion inclusive functionals estimate a $20-40 \mathrm{meV}$ stronger interaction for the water/boronene and water/h-BN systems compared to reference interaction energies. As such, optB86b-vdW and vdW-DF2 predict the wrong trend for the adsorption energy from zero to 2D systems, overestimating the adsorption energy at the minimum by up to $40 \%$ on the larger BN substrates. Indeed, a similar behavior has been recently seen for water inside a carbon nanotube. ${ }^{120}$ In Sec. III C, we will elaborate on this overestimation.

Dispersion interactions can also be accounted for in DFT calculations with dispersion corrections to $x c$ functionals, such as in $\mathrm{PBE}+\mathrm{D} 3$. Although $\mathrm{PBE}+\mathrm{D} 3$ can be seen to overestimate the interaction energy of water on all of the BN substrates in Fig. 2, it predicts, in agreement with the reference methods, that the adsorption energy does not increase from zero to 2D. Let us also consider the MBD correction which has been evaluated in combination with PBE for different supercells in Table II, and the interaction energy curves are shown in Fig. 2. In this way, the periodic dipole potential is summed over a long distance, and all possible collective charge density fluctuations are converged. Indeed, PBE+MBD captures the same higher-order, non-additive correlation interactions as the RPA. However, the MBD correction with PBE leads to 15\%-35\% overestimation of the interaction energy on the molecular and surface substrates, compared to wavefunction based correlated methods.

The best performance amongst the $x c$ functionals we have considered is given by the recently developed SCAN functional. SCAN predicts the water/borazine interaction energy to be $-126 \mathrm{meV}$ at a distance of $3.22 \AA$, and it agrees perfectly with DMC at water-borazine separations above $3.5 \AA$. In the absence of any dispersion correction to SCAN, the interaction energy at larger water-borazine separation distances reflects the LDA-like construction of this functional and the inclusion of some long-range correlation energy that was incorporated using an Ar dimer interaction. ${ }^{44}$ SCAN also predicts a $15 \mathrm{meV}$ weaker interaction for water/boronene compared to water/borazine-in line with the trends given by LRDMC, DMC, LCCSD(T), and MP2. However, SCAN predicts interaction energies in close agreement with the reference interaction energies of water/boronene and water/h-BN, despite the absence of any vdW correction. Thus, the implementation of any vdW-correction to SCAN has to be done cautiously in order to avoid considerable errors. Still, it has been shown that many properties derived by SCAN can be improved by incorporating a long-range dispersion correction. ${ }^{121}$ For a detailed analysis of the performance of a selection of other widely used $x c$ functionals on the h-BN surface, we refer the interested reader to Ref. 19.

In this section, we have seen that various DFT $x c$ functionals need further improvement, and the challenge arises in the larger systems for which the inclusion of vdW interactions leads to an overestimation of the interaction energy. The likely sources of error in the $x c$ functionals are discussed in Sec. III C.

\section{Static and dynamic polarizabilities of the water/BN interactions from TD-DFT}

The adsorption of water on $\mathrm{BN}$ substrates of different sizes and dimensions is found to have almost the same adsorption energy, as demonstrated in Fig. 4(a). This is contrary to the naive expectation that the adsorption energy increases with the size of the system due to increased dispersion interaction. The expected behavior of the water adsorption energy is given instead by vdW models such as optB86b-vdW and vdW-DF2, also shown in Fig. 4(a). These methods predict a monotonic increase in the adsorption energy of water with the increasing size of the BN substrate. In order to understand this outcome better, the static and dynamic polarlizabilities of each system have been computed. These polarizabilities are observable and therefore provide information that is useful to both computational and experimental studies. The static polarizability $\alpha_{k l}^{0}$ of a system describes its response to external fields giving rise to the induction energy. A change in the induction energy will be mainly determined by the static polarizability of the BN systems. We report the in-plane and out-of-plane polarizabilities $\alpha_{\|}^{0}$ and $\alpha_{\perp}^{0}$, respectively, where the latter is particularly relevant for the present adsorption geometries. The dynamic polarizability $\alpha_{k l}(i \omega)$, on the other hand, determines the dispersion interaction in a system; its leading order can be described by molecular $\mathrm{C}_{6}^{\mathrm{AB}}$ dispersion coefficients. ${ }^{122,123}$ We present these intermolecu$\operatorname{lar} \mathrm{C}_{6}^{\mathrm{AB}}$ coefficients for the interaction between water and the different $\mathrm{BN}$ systems. A common approximation is the use of isotropic dynamic polarizabilities $\left(\alpha_{\mathrm{iso}}=\frac{1}{3} \operatorname{Tr}\left[\alpha_{\mathrm{k}}\right]\right)$. This results in an isotropic dispersion coefficient $\mathrm{C}_{6}^{\text {iso }}$, which we contrast with $\mathrm{C}_{6}^{\text {full }}$ arising from the full polarizability tensor. Note that the above description of induction and dispersion interactions is only valid in the long-range (i.e., well separated) limit.

The molecular polarizabilities $\alpha$ have been computed from TD-DFT with well established numerical settings (PBE38 functional in the def2-QZVPD-aug basis, solving a non-standard eigenvalue problem in the frequency domain). ${ }^{42,124} \mathrm{~A}$ comparison to the MBD model 


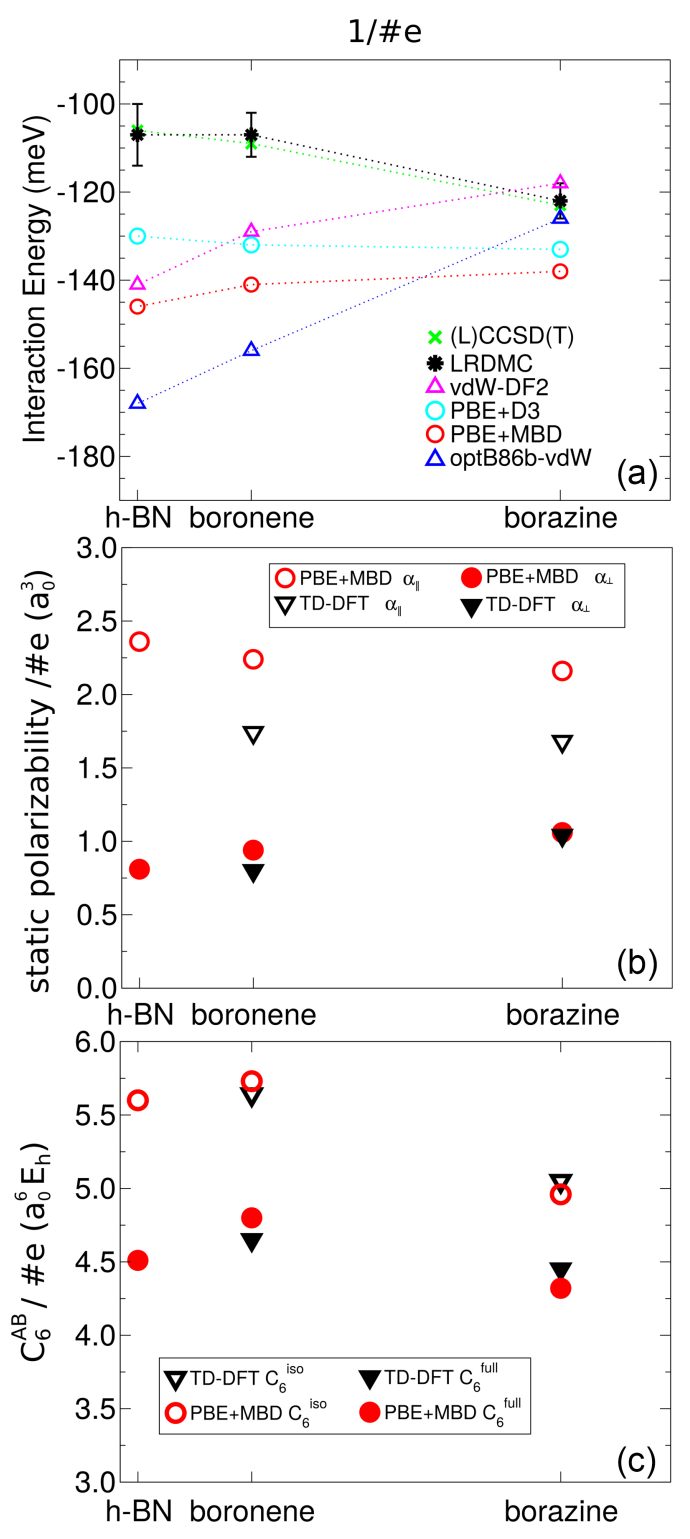

FIG. 4. Observable properties of water/BN systems. (a) Adsorption energy in meV of water on BN substrates: h-BN with a $(4 \times 4)$ unit cell, boronene, and borazine. (b) Static polarizability normalised for the total number of electrons in the BN substrate, from TD-DFT (black triangles) and PBE+MBD (red circles). Empty symbols indicate the in-plane static polarizability $\alpha_{\|}^{0}$. Filled symbols indicate the out-of-plane static polarizability $\alpha_{\perp}^{0}$. (c) Molecular $\mathrm{C}_{6}^{\text {iso }}$ and $\mathrm{C}_{6}^{\text {full }}$ per electron with respect to the $\mathrm{BN}$ substrate, from TD-DFT (black triangles) and PBE+MBD (red circles). $\mathrm{C}_{6}^{\text {iso }}$ is the isotropic dynamic polarizability and is indicated by empty symbols. $\mathrm{C}_{6}^{\text {full }}$ is calculated from the full dynamic polarizability tensor accounting for anisotropy and is indicated by filled symbols.

polarizabilities is shown in Fig. 4. Details of the TD-DFT calculations and the polarizability evaluations can be found in the supplementary material along with the structures of the water/BN complexes.

Let us start by considering the static polarizabilities. Figure 4(b) shows that $\alpha_{\|}^{0}$ is significantly higher than $\alpha_{\perp}^{0}$ on all BN substrates and also invariant with respect to the substrate. However, $\alpha_{\perp}^{0}$ is reduced from borazine to boronene. As such, anisotropy in the polarizability is clearly important in this system, not only resulting in a reduced induction energy per electron for each water/BN system but also changing the relative trend. The neglect of anisotropy leads to an overestimation of the static polarizability, indicating that the induction interaction with water is also overestimated. Note that this form of anisotropy (or non-additivity) is captured at the canonical HF level of theory, as well as by $x c$ functionals in DFT. However, it is not given that the absolute value of the static polarizability is accurate within either of these approaches and is unlikely to be captured by classical force fields.

Second, the effective molecular $\mathrm{C}_{6}^{\mathrm{AB}}$ coefficients have been computed for water/borazine and water/boronene from TD-DFT. The TD-DFT results show that $\mathrm{C}_{6}^{\text {full }}$ is $14 \%$ smaller than $\mathrm{C}_{6}^{\text {iso }}$ for water/borazine and $21 \%$ smaller for water/boronene. Once again, this suggests that anisotropy in the polarizability plays a key role in the dispersion interaction such that the dispersion energy is overestimated in the isotropic case. Indeed, in Fig. 4(a), the predicted adsorption energies on boronene and h-BN from optB86b-vdW and vdW-DF2 demonstrate that an isotropic model of $\mathrm{vdW}$ interactions leads to a significantly larger estimation of the adsorption energy of water.

PBE+MBD as well as PBE+D3 have been found to overestimate the interaction energies despite reproducing the stability of the water adsorption energy from zero to $2 \mathrm{D}$. The effective molecular $\mathrm{C}_{6}^{\mathrm{AB}}$ coefficients are used to assess the accuracy of MBD for predicting the dispersion contribution to the interaction energies in the molecular adsorption systems. In fact, we can see from Table III that MBD accurately predicts $\mathrm{C}_{6}^{\text {full }}$ within $3 \%$ of the reference TD-DFT for water/borazine and water/boronene, and we can thus trust the water/h-BN results. The static polarizability for h-BN is comparable, though slightly smaller than for boronene. Although the absolute $\mathrm{C}_{6}^{\mathrm{AB}}$ coefficient is also larger on h-BN than on boronene, after normalising for the number of electrons, $\mathrm{C}_{6}^{\text {full }}$ appears slightly smaller on the extended BN surface. The small difference can be attributed to the lack of hydrogen atoms in the h-BN surface, which contributes to $\mathrm{C}_{6}^{\text {full }}$ in boronene. As the long-range limit in PBE-MBD seems to be well captured, the short to medium range interaction is more likely to be a source of error and, in particular, the interface between MBD and the underlying $x c$ functional.

To summarize the findings in this section, molecular polarizabilities of water/borazine, water/boronene, and water/hBN systems have been computed using TD-DFT and MBD. The results of the static polarizability have indicated that the

TABLE III. Reference information for the polarizabilities of water/BN systems: In-plane $\left(\alpha_{\|}^{0}\right)$ and out-of-plane $\left(\alpha_{\perp}^{0}\right)$ static polarizabilities $\left[a_{0}^{3}\right]$, and isotropic $\left(\mathrm{C}_{6}^{\text {iso }}\right)$ and full $\left(\mathrm{C}_{6}^{\text {full }}\right)$ dispersion coefficients $\left[E_{h} a_{0}^{6}\right]$ from TD-DFT and PBE+MBD. All values are given per electron and $a_{0}$ is Bohr's radius.

\begin{tabular}{lcccc}
\hline \hline & $\alpha_{\|}^{0}$ & $\alpha_{\perp}^{0}$ & $\mathrm{C}_{6}^{\text {iso }}$ & $\mathrm{C}_{6}^{\text {full }}$ \\
\hline TD-DFT & & & & \\
Borazine & 1.68 & 1.04 & 5.05 & 4.45 \\
Boronene & 1.74 & 0.80 & 5.64 & 4.65 \\
PBE+MBD & & & & \\
Borazine & 2.16 & 1.06 & 4.96 & 4.32 \\
$\begin{array}{l}\text { Boronene } \\
\text { h-BN }\end{array}$ & 2.24 & 0.94 & 5.73 & 4.80 \\
\hline \hline
\end{tabular}


induction energy per electron decreases from the small molecular borazine system to the larger boronene molecule and to the extended h-BN surface. At the same time, it is countered by the increase in the dispersion interaction, resulting in almost the same adsorption energy of water on these BN substrates that span zero to 2-dimensions. Furthermore, the anisotropy in the polarizability of these systems is significant and cannot be captured using isotropic dispersion coefficients or standard vdW models.

\section{CONCLUSION}

The adsorption energy of water on $\mathrm{BN}$ substrates has been determined from coupled cluster theory, LRDMC, DMC, MP2, and RPA based methods. The best estimate of the water/h-BN adsorption energy is $-107 \pm 7 \mathrm{meV}$ from LRDMC. Various corrections have been quantified and the most significant include single excitations to the RPA, perturbative triple excitations in $\operatorname{CCSD}(\mathrm{T})$, and the contribution from long range correlation energy on the h-BN surface. Each was found to contribute $\sim 20 \mathrm{meV}$ to the adsorption energy of water. We report static polarizabilities and effective $\mathrm{C}_{6}^{\mathrm{AB}}$ dispersion coefficients from TD-DFT and MBD. Interestingly, a significant amount of non-additivity is found in the dispersion interaction of water with h-BN, despite the substrate being a wide band gap insulating system. The non-additive interaction in these systems is due to the high degree of anisotropy. The findings show that the MBD correction is a promising method for estimating the long-range correlation contribution especially for highly anisotropic, low-dimensional structures. This is particularly useful for more expensive many-body wavefunction based periodic methods, in which some of the inherent finite size effects can be estimated by the MBD method in future. However, it is clear for the $x c$ functionals considered here that there is still a lot of scope for improvementparticularly in the development of $x c$ functionals for the accurate prediction of adsorption energies. The adsorption energies, static polarizabilities, and molecular dispersion coefficients presented in this study also provide an opportunity for future experimental measurements of these properties to be compared.

\section{SUPPLEMENTARY MATERIAL}

See supplementary material for a detailed description of static and dynamic polarizability calculations as well as the geometries of the benchmark water/BN configurations.

\section{ACKNOWLEDGMENTS}

We are grateful for the support from University College London and Argonne National Laboratory (ANL) through the Thomas Young Centre-ANL initiative. Some of the research leading to these results has received funding from the European Research Council under the European Union's Seventh Framework Programme (No. FP/2007-2013)/ERC Grant Agreement No. 616121 (HeteroIce project). J.G.B. acknowledges the support by the Humboldt Foundation within the Feodor-Lynen program. A.M. is supported by the Royal Society through a Wolfson Research Merit Award. This research used resources of the Argonne Leadership Computing Facility at Argonne National Laboratory, which is supported by the Office of Science of the U.S. DOE under Contract No. DEAC02-06CH11357. This research also used resources as part of an INCITE project (awarded to D.A.) at the Oak Ridge National Laboratory (Rhea/Eos), which is supported by the Office of Science of the U.S. Department of Energy (DOE) under Contract No. DEAC05-00OR22725. In addition, we are grateful for computing resources provided by the London Centre for Nanotechnology and research computing at University College London. M.R. acknowledges the support from the University of Oxford and the École Polytechnique Fédérale de Lausanne, as well as funding from the Otto Hahn Award of the Max Planck Society. B.R. and G.K. acknowledge funding by the Austrian Science Fund (FWF) F41 (SFB ViCoM). Computations were performed on the Vienna Scientific Cluster VSC3.

${ }^{1}$ K. Autumn, M. Sitti, Y. A. Liang, A. M. Peattie, W. R. Hansen, S. Sponberg, T. W. Kenny, R. Fearing, J. N. Israelachvili, and R. J. Full, Proc. Natl. Acad. Sci. U. S. A. 99, 12252 (2002).

${ }^{2}$ A. Y. Stark, T. W. Sullivan, and P. H. Niewiarowski, J. Exp. Biol. 215, 3080 (2012).

${ }^{3}$ A. Y. Stark, I. Badge, N. A. Wucinich, T. W. Sullivan, P. H. Niewiarowski, and A. Dhinojwala, Proc. Natl. Acad. Sci. U. S. A. 110, 6340 (2013).

${ }^{4}$ I. Badge, A. Y. Stark, E. L. Paoloni, P. H. Niewiarowski, and A. Dhinojwala, Sci. Rep. 4, 6643 (2014).

${ }^{5}$ V. V. Gobre and A. Tkatchenko, Nat. Commun. 4, 2341 (2013).

${ }^{6}$ W. Lei, D. Portehault, D. Liu, S. Qin, and Y. Chen, Nat. Commun. 4, 1777 (2013).

${ }^{7}$ M. Yusuf, F. M. Elfghi, S. A. Zaidi, E. C. Abdullah, and M. A. Khan, RSC Adv. 5, 50392 (2015).

${ }^{8}$ S. L. Price, Chem. Soc. Rev. 43, 2098 (2014).

${ }^{9}$ W. A. Brown, R. Kose, and D. A. King, Chem. Rev. 98, 797 (1998).

${ }^{10}$ C. T. Campbell and J. R. V. Sellers, Chem. Rev. 113, 4106 (2013).

${ }^{11}$ L. Schimka, J. Harl, A. Stroppa, A. Grüneis, M. Marsman, F. Mittendorfer, and G. Kresse, Nat. Mater. 9, 741 (2010).

${ }^{12}$ S. J. Binnie, S. J. Nolan, N. D. Drummond, D. Alfè, N. L. Allan, F. R. Manby, and M. J. Gillan, Phys. Rev. B 82, 165431 (2010).

${ }^{13}$ T. Tsatsoulis, F. Hummel, D. Usvyat, M. Schütz, G. H. Booth, S. S. Binnie, M. J. Gillan, D. Alfè, A. Michaelides, and A. Grüneis, J. Chem. Phys. 146, 204108 (2017).

${ }^{14}$ J. Ma, A. Michaelides, D. Alfè, L. Schimka, G. Kresse, and E. Wang, Phys. Rev. B 84, 033402 (2011).

${ }^{15}$ E. Voloshina, D. Usvyat, M. Schütz, Y. Dedkov, and B. Paulus, Phys. Chem. Chem. Phys. 13, 12041 (2011).

${ }^{16}$ P. Beate and K. Rosciszewski, Int. J. Quantum Chem. 109, 3055 (2009).

${ }^{17}$ C. Müller, B. Herschend, K. Hermansson, and B. Paulus, J. Chem. Phys. 128, 214701 (2008).

${ }^{18}$ C. Müller, B. Paulus, and K. Hermansson, Surf. Sci. 603, 2619 (2009).

${ }^{19}$ Y. S. Al-Hamdani, M. Ma, D. Alfè, O. A. von Lilienfeld, and A. Michaelides, J. Chem. Phys. 142, 181101 (2015).

${ }^{20}$ P. Ganesh, J. Kim, C. Park, M. Yoon, F. A. Reboredo, and P. R. C. Kent, J. Chem. Theory Comput. 10, 5318 (2014).

${ }^{21}$ M. C. Gordillo, Phys. Rev. B 88, 041406 (2013).

${ }^{22}$ S. Lei, B. Paulus, S. Li, and B. Schmidt, J. Comput. Chem. 37, 1313 (2016).

${ }^{23}$ J. Ma, A. Michaelides, and D. Alfè, J. Chem. Phys. 134, 134701 (2011).

${ }^{24}$ S. J. Binnie, "Ab initio surface energetics: Beyond chemical accuracy," Ph.D. thesis, University College London, 2011.

${ }^{25}$ Y. Wu, L. K. Wagner, and N. R. Aluru, J. Chem. Phys. 142, 234702 (2015).

${ }^{26}$ Y. Wu, L. K. Wagner, and N. R. Aluru, J. Chem. Phys. 144, 164118 (2016).

${ }^{27}$ G. R. Jenness, O. Karalti, and K. D. Jordan, Phys. Chem. Chem. Phys. 12, 6375 (2010).

${ }^{28}$ A. Zen, L. M. Roch, S. J. Cox, X. L. Hu, S. Sorella, D. Alfè, and A. Michaelides, J. Phys. Chem. C 120, 26402 (2016).

${ }^{29} \mathrm{We}$ refer to this molecule as boronene in this study for convenience.

${ }^{30}$ A. Ambrosetti, N. Ferri, R. A. DiStasio, and A. Tkatchenko, Science 351, 1171 (2016).

${ }^{31}$ A. Ambrosetti, A. M. Reilly, R. A. DiStasio, and A. Tkatchenko, J. Chem. Phys. 140, 18A508 (2014). 
${ }^{32}$ A. Tkatchenko, A. Ambrosetti, and R. A. Distasio, J. Chem. Phys. 138, 074106 (2013).

${ }^{33}$ The residual interaction of water with h-BN at $8 \AA$ distance is less than 4 meV.

${ }^{34}$ A. Bosak, J. Serrano, M. Krisch, K. Watanabe, T. Taniguchi, and H. Kanda, Phys. Rev. B 73, 041402 (2006).

${ }^{35}$ G. Kresse, Phys. Rev. B 59, 1758 (1999).

${ }^{36}$ P. E. Blöchl, Phys. Rev. B 50, 17953 (1994).

${ }^{37}$ J. P. Perdew, K. Burke, and M. Ernzerhof, Phys. Rev. Lett. 77, 3865 (1996).

${ }^{38}$ C. Adamo and V. Barone, J. Chem. Phys. 110, 6158 (1999).

${ }^{39}$ J. P. Perdew, M. Ernzerhof, and K. Burke, J. Chem. Phys. 105, 9982 (1996).

${ }^{40}$ J. Klimeš, D. R. Bowler, and A. Michaelides, Phys. Rev. B 83, 195131 (2011).

${ }^{41}$ A. D. Becke, J. Chem. Phys. 84, 4524 (1986).

${ }^{42}$ S. Grimme, J. Antony, S. Ehrlich, and H. Krieg, J. Chem. Phys. 132, 154104 (2010).

${ }^{43}$ S. Grimme, S. Ehrlich, and L. Goerigk, J. Comput. Chem. 32, 1456 (2011).

${ }^{44}$ J. Sun, A. Ruzsinszky, and J. Perdew, Phys. Rev. Lett. 115, 036402 (2015).

${ }^{45}$ V. Blum, R. Gehrke, F. Hanke, P. Havu, V. Havu, X. Ren, K. Reuter, and M. Scheffler, Comput. Phys. Commun. 180, 2175 (2009).

${ }^{46}$ M. J. Frisch, G. W. Trucks, H. B. Schlegel, G. E. Scuseria, M. A. Robb, J. R. Cheeseman, J. A. Montgomery, Jr., T. Vreven, K. N. Kudin, J. C. Burant, J. M. Millam et al., Gaussian 03, Revision C.02, Gaussian, Inc., 2003.

${ }^{47}$ H. J. Werner, P. J. Knowles, G. Knizia, and F. R. Manby, MoLPro, version 2012.1, a package of ab initio programs, 2012, see http://www.molpro.net.

${ }^{48}$ M. Valiev, E. J. Bylaska, N. Govind, K. Kowalski, T. P. Straatsma, H. J. Van Dam, D. Wang, J. Nieplocha, E. Apra, and T. Windus, Comput. Phys. Commun. 181, 1477 (2010).

${ }^{49}$ D. G. Liakos and F. Neese, J. Phys. Chem. A 116, 4801 (2012)

${ }^{50}$ T. H. Dunning, J. Chem. Phys. 90, 1007 (1989).

${ }^{51}$ D. Feller, J. Comput. Chem. 17, 1571 (1996).

${ }^{52}$ C. Riplinger, B. Sandhoefer, A. Hansen, and F. Neese, J. Chem. Phys. 139, 134101 (2013).

${ }^{53}$ F. Neese, Wiley Interdiscip. Rev.: Comput. Mol. Sci. 2, 73 (2012).

${ }^{54}$ C. Riplinger, P. Pinski, U. Becker, E. F. Valeev, and F. Neese, J. Chem. Phys. 144, 024109 (2016).

${ }^{55}$ D. G. Liakos, M. Sparta, M. K. Kesharwani, J. M. L. Martin, and F. Neese, J. Chem. Theory Comput. 11, 1525 (2015).

${ }^{56}$ M. Marsman, A. Grüneis, J. Paier, and G. Kresse, J. Chem. Phys. 130, 184103 (2010).

${ }^{57}$ A. Grüneis, M. Marsman, J. Harl, L. Schimka, and G. Kresse, J. Chem. Phys. 131, 154115 (2009).

${ }^{58}$ M. Kaltak, J. Klimeš, and G. Kresse, Phys. Rev. B 90, 054115 (2014).

${ }^{59}$ M. Kaltak, J. Klimeš, and G. Kresse, J. Chem. Theory Comput. 10, 2498 (2014).

${ }^{60}$ J. Klimeš, M. Kaltak, E. Maggio, and G. Kresse, J. Chem. Phys. 143, 102816 (2015).

${ }^{61}$ G. H. Booth, T. Tsatsoulis, G. K.-L. Chan, and A. Grüneis, J. Chem. Phys. 145, 084111 (2016).

${ }^{62}$ A. Grüneis, M. Marsman, and G. Kresse, J. Chem. Phys. 133, 074107 (2010).

${ }^{63}$ A. Grüneis, Phys. Rev. Lett. 115, 066402 (2015).

${ }^{64}$ T. Schäfer, B. Ramberger, and G. Kresse, J. Chem. Phys. 146, 104101 (2017).

${ }^{65}$ Differently to current default settings in VASP, NOMEGA $=8$ was set and PRECFOCK $=$ Normal for the RPA calculations .

${ }^{66}$ J. Harl and G. Kresse, Phys. Rev. B 77, 045136 (2008).

${ }^{67}$ J. Klimeš, M. Kaltak, and G. Kresse, Phys. Rev. B 90, 075125 (2014).

${ }^{68}$ For a $(4 \times 4)$ h-BN unit cell, the cubic scaling implementation predicts adsorption energies of $-65 \mathrm{meV}$, whereas the basis set extrapolated PGTO value is $-69 \mathrm{meV}$. Increasing the cutoff from $430 \mathrm{eV}$ to $500 \mathrm{eV}$ in the cubic scaling code does not change these values. The slight differences are most likely related to different basis set extrapolation procedures.

${ }^{69}$ W. M. C. Foulkes, L. Mitas, R. J. Needs, and G. Rajagopal, Rev. Mod. Phys. 73, 33 (2001)

${ }^{70}$ M. Casula, C. Filippi, and S. Sorella, Phys. Rev. Lett. 95, 100201 (2005).

${ }^{71}$ M. Casula, S. Moroni, S. Sorella, and C. Filippi, J. Chem. Phys. 132, 154113 (2010).

${ }^{72}$ P. J. Reynolds, D. M. Ceperley, B. J. Alder, and W. A. Lester, J. Chem. Phys. 77, 5593 (1982).

${ }^{73}$ A. Zen, S. Sorella, M. J. Gillan, A. Michaelides, and D. Alfè, Phys. Rev. B 93, 241118(R) (2016)
${ }^{74}$ R. J. Needs, M. D. Towler, N. D. Drummond, and P. López Ríos, J. Phys. Condens. Matter 22, 023201 (2010).

${ }^{75}$ J. R. Trail and R. J. Needs, J. Chem. Phys. 122, 174109 (2005).

${ }^{76}$ J. R. Trail and R. J. Needs, J. Chem. Phys. 122, 014112 (2005).

${ }^{77}$ Y. S. Al-Hamdani, D. Alfè, O. A. von Lilienfeld, and A. Michaelides, J. Chem. Phys. 141, 18C530 (2014).

${ }^{78}$ S. Baroni, A. Dal Corso, S. de Gironcoli, and P. Giannozzi, http://www. pwscf.org, access date: 1 June 2015.

${ }^{79}$ D. Alfè and M. J. Gillan, Phys. Rev. B 70, 161101(R) (2004).

${ }^{80}$ J. P. Perdew and A. Zunger, Phys. Rev. B 23, 5048 (1981).

${ }^{81}$ B. Santra, J. Klimeš, D. Alfè, A. Tkatchenko, B. Slater, A. Michaelides, R. Car, and M. Scheffler, Phys. Rev. Lett. 107, 185701 (2011).

${ }^{82}$ B. Santra, J. Klimeš, A. Tkatchenko, D. Alfè, B. Slater, A. Michaelides, R. Car, and M. Scheffler, J. Chem. Phys. 139, 154702 (2013).

${ }^{83}$ D. Quigley, D. Alfè, and B. Slater, J. Chem. Phys. 141, 161102 (2014).

${ }^{84}$ M. A. Morales, J. R. Gergely, J. McMinis, J. M. McMahon, J. Kim, and D. M. Ceperley, J. Chem. Theory Comput. 10, 2355 (2014).

${ }^{85}$ S. J. Cox, M. D. Towler, D. Alfè, and A. Michaelides, J. Chem. Phys. 140, 174703 (2014)

${ }^{86}$ A. Benali, L. Shulenburger, N. A. Romero, J. Kim, and O. A. von Lilienfeld, J. Chem. Theory Comput. 10, 3417 (2014).

${ }^{87}$ M. J. Gillan, D. Alfè, and F. R. Manby, J. Chem. Phys. 143, 102812 (2015).

${ }^{88}$ M. Morales, R. Clay, C. Pierleoni, and D. Ceperley, Entropy 16, 287 (2014).

${ }^{89}$ M. Dubecký, L. Mitas, and P. Jurečka, Chem. Rev. 116, 5188 (2016).

${ }^{90}$ A. Zen, Y. Luo, G. Mazzola, L. Guidoni, and S. Sorella, J. Chem. Phys. 142, 144111 (2015).

${ }^{91}$ J. Chen, A. Zen, J. G. Brandenburg, D. Alfè, and A. Michaelides, Phys. Rev. B 94, 220102 (2016).

${ }^{92}$ L. Mitas, E. L. Shirley, and D. Ceperley, J. Chem. Phys. 95, 3467 (1991).

${ }^{93}$ Note that in the time this work has been conducted, Zen et al. implemented a more efficient method in the CASINO code that allows for better convergence with large time steps. ${ }^{73}$ We tested the water/boronene system with this new implementation for time steps of 0.050 a.u. (considered as $\tau_{\text {standard }}$ with the new implementation) and 0.025 a.u. (considered as $\tau_{\text {small }}$ ). The water/boronene interactions are converged within the stochastic error for both implementations and time steps in CASINO.

${ }^{94}$ S. Sorella and L. Capriotti, Phys. Rev. B 61, 2599 (2000).

${ }^{95}$ M. Buonaura and S. Sorella, Phys. Rev. B 57, 11446 (1998).

${ }^{96}$ LRDMC $^{70,71}$ preserves the variational principle even when used in combination with nonlocal pseudopotentials, and it is size-consistent for any value of the mesh $a$, maintaining its efficiency even for systems with a large number of electrons.

${ }^{97}$ The TurboRVB Quantum Monte Carlo package includes a complete suite for variational, diffusion, and Green function quantum Monte Carlo calculations on molecules and solids, for wave function and geometry optimization and for quantum Monte Carlo based molecular dynamics simulations. It is developed by Sorella and co-workers, http://people.sissa.it/ sorella/web/index.html, access date: 1 June 2015.

${ }^{98}$ A. Zen, Y. Luo, S. Sorella, and L. Guidoni, J. Chem. Theory Comput. 9 , 4332 (2013).

${ }^{99} \mathrm{We}$ used a $(7 s, 2 p)$ basis for the $\mathrm{H}$ atoms, $(9 s, 10 p, 2 d)$ for $\mathrm{B},(10 s, 10 p$, $2 d$ ) for $\mathrm{N}$, and $(10 s, 11 p, 2 d)$ for $\mathrm{O}$, all of them with Gaussian-type atomic orbitals. Note that, due to the presence of the Jastrow factor, the orbitals with too high value for the exponent or with very high angular momentum lead to an almost negligible improvement in the variational wave function quality, in addition to a much slower and inefficient optimisation of the parameters. ${ }^{98}$ Thus, the initial $\mathrm{B}, \mathrm{N}$, and $\mathrm{O}$ exponents have been taken starting from the Burkatzki et al. VTZ basis set ${ }^{125}$ for which we have uncontracted and removed the $f$ orbitals and the largest exponents (i.e., $>30$ ) or the near redundant ones. For $\mathrm{H}$, which has no pseudopotential, we used an all-electron basis set.

${ }^{100}$ M. Burkatzki, C. Filippi, and M. Dolg, J. Chem. Phys. 126, 234105 (2007).

${ }^{101}$ S. Azadi, C. Cavazzoni, and S. Sorella, Phys. Rev. B 82, 125112 (2010).

${ }^{102}$ The non-homogeneous terms are expressed in terms of atomic orbitals, which are expanded in terms of a $(2 s, 2 p)$ basis for $\mathrm{H}$ atoms, $(3 s, 2 p, 1 d)$ for $\mathrm{B}, \mathrm{N}$, and $\mathrm{O}$ atoms.

${ }^{103}$ A. Zen, B. L. Trout, and L. Guidoni, J. Chem. Phys. 141, 014305 (2014).

${ }^{104}$ A. Zen, E. Coccia, Y. Luo, S. Sorella, and L. Guidoni, J. Chem. Theory Comput. 10, 1048 (2014).

${ }^{105}$ A. Zen, E. Coccia, S. Gozem, M. Olivucci, and L. Guidoni, J. Chem. Theory Comput. 11, 992 (2015).

${ }^{106}$ N. D. Drummond, R. J. Needs, A. Sorouri, and W. M. C. Foulkes, Phys. Rev. B 78, 125106 (2008). 
${ }^{107}$ M. Holzmann, R. C. Clay, M. A. Morales, N. M. Tubman, D. M. Ceperley, and C. Pierleoni, Phys. Rev. B 94, 035126 (2016).

${ }^{108}$ L. M. Fraser, W. M. C. Foulkes, G. Rajagopal, R. J. Needs, S. D. Kenny, and A. J. Williamson, Phys. Rev. B 53, 1814 (1996).

${ }^{109}$ A. J. Williamson, G. Rajagopal, R. J. Needs, L. M. Fraser, W. M. C. Foulkes, Y. Wang, and M.-Y. Chou, Phys. Rev. B 55, R4851 (1997).

${ }^{110}$ P. R. C. Kent, R. Q. Hood, A. J. Williamson, R. J. Needs, W. M. C. Foulkes, and G. Rajagopal, Phys. Rev. B 59, 1917 (1999).

${ }^{111}$ S. Chiesa, D. M. Ceperley, R. M. Martin, and M. Holzmann, Phys. Rev. Lett. 97, 076404 (2006).

${ }^{112}$ H. Kwee, S. Zhang, and H. Krakauer, Phys. Rev. Lett. 100, 126404 (2008).

${ }^{113}$ S. Azadi and W. M. C. Foulkes, J. Chem. Phys. 143, 102807 (2015).

${ }^{114}$ It should be noted that without CBS extrapolation, there is a $20 \mathrm{meV}$ overestimation for the MP2 energy with Dunning's aug-cc-pVTZ basis set, and it is therefore essential to employ basis set extrapolations.

${ }^{115}$ X. Ren, A. Tkatchenko, P. Rinke, and M. Scheffler, Phys. Rev. Lett. 106, 153003 (2011).

${ }^{116}$ J. Hermann, D. Alfè, A. Tkatchenko, H. Krieg, and R. J. Needs, Nat. Commun. 8, 14052 (2017).

${ }^{117}$ After checking convergence, a $500 \mathrm{eV}$ energy cutoff was employed for the one-particle HF states along with $\Gamma$-point sampling of the Brillouin zone. The cutoff energy for the auxiliary plane-wave basis set required for the evaluation of the two-electron four-orbital integrals ${ }^{56}$ was set to 250 eV. Occupied HF states were converged within the full plane-wave basis, whereas the virtual orbitals were constructed using Dunning's contracted aug-cc-pVDZ and aug-cc-pVTZ ${ }^{50,51}$ pseudized Gaussians in a plane-wave representation, projected onto the HF occupied states.

${ }^{118}$ K. Liao and A. Grüneis, J. Chem. Phys. 145, 141102 (2016).

${ }^{119}$ T. Gruber, K. Liao, F. Hummel, T. Tsatsoulis, and A. Grüneis, "Applying the gold-standard of quantum chemistry to solids and surfaces in the thermodynamic limit" (unpublished).

${ }^{120}$ Y. S. Al-Hamdani, D. Alfè, and A. Michaelides, J. Chem. Phys. 146, 094701 (2017).

${ }^{121}$ J. G. Brandenburg, J. E. Bates, J. Sun, and J. P. Perdew, Phys. Rev. B 94, 115144 (2016).

${ }^{122}$ A. J. Stone, The Theory of Intermolecular Forces (Oxford University Press, Oxford, 1997).

${ }^{123}$ H. B. G. Casimir and D. Polder, Phys. Rev. 73, 360 (1948).

${ }^{124}$ M. E. Casida, in Recent Advances in Density Functional Methods, edited by D. P. Chong (World Scientific, Singapore, 1995), Vol. 1, pp. 155-192.

${ }^{125}$ See http://burkatzki.com/pseudos/index.2.html for an interface providing access to the pseudopotentials and the accompanying basis sets presented in Ref. 100, access date: 1 March 2015. 